\title{
Cross-Cultural Comparison of Early Maladaptive Schemas, Resilience and Quality of Life in Students
}

\author{
Sogand Bakhtiari Moghaddam ${ }^{1} \&$ Farhad Jomehri ${ }^{2}$ \\ ${ }^{1}$ Department of Psychology, Islamic Azad University, Qazvin Branch, Qazvin, Iran \\ ${ }^{2}$ Department of psychology, Allameh Tabataba'i University, Tehran, Iran \\ Correspondence: Farhad Jomehri, Department of psychology, Allameh Tabataba'i University, Tehran, \\ 1489684511, Iran. Tel: 98-2144-7375-1019. E-mail: farhadjomehri@yahoo.com
}

Received: April 4, 2016

Accepted: April 28, 2016

Online Published: May 22, 2016

doi:10.5539/res.v8n2p236

URL: http://dx.doi.org/10.5539/res.v8n2p236

\begin{abstract}
This study compared early maladaptive schemas, resilience, and quality of life of Iranian and German students. This was a descriptive causal-comparative study. To this end, 100 German students and 100 Iranian students were selected using the cluster random sampling method and were evaluated using the research instruments. The instruments used included demographic questionnaire, and Yang's Schema Questionnaire, the World Health Organization's Quality of Life Questionnaire and Connor-Davidson's Resilience Scale that are common instruments in psychology and their validity and reliability have been confirmed in various studies. Research data using multivariate analysis of variance and independent t-test were analyzed. Statistical analysis of the research hypotheses showed a significant difference in the early maladaptive schemas of Iranian and German students $(p<0.05)$. However, there was no difference between resilience and quality of life of the Iranian and German students $(\mathrm{p}<0.05)$.
\end{abstract}

Keywords: resilience, early maladaptive schemas, quality of life, Iranian students, german students

\section{Introduction}

The world, in which we live, compared with three or four decades ago has changed into a place where ethnicities, nationalities, religions, races and languages and generally cultures and subcultures live in peace or in conflict. This may increasingly lead to the spread of psychological injuries. The spread of global communication tools such as mobile phones, computers and the Internet, formation of working groups composed of experts in various fields of science and technology, expansion of scientific and academic exchanges, on one hand and the spread of international terrorism, superpowers campaigns and the jeopardizing the world peace and so on have positively or negatively led to the peace or conflict in the different cultural groups (Yazdkhasti et al., 2011).

Culture, is a broad but simple concept that includes (overt and covert) cognitive patterns and their realization in social institutions, activities and built by handicrafts, distinguishing one group from the other groups (Asgari \& Roshani, 2012). Although culture and well-being, as well as other cultural factors such as individualism and collectivism have been investigated in most of the studies, the mechanisms of mental health and psychological variables of the different cultures have been less considered. This study seeks to investigate the role of the variables such as quality of life, early maladaptive schemas, and resilience in the form of a cross-cultural study.

The early maladaptive schemas are the first variable that is investigated in two different cultures. The word "schema" in psychology, has a rich and outstanding history. In cognitive development, schema is formed based on reality or experience to help people shape their own experiences. Moreover, the perception is mediated through the scheme and all answers are directed by the schemas; therefore, schema is an abstract manifestation of the distinguishing characteristics of an event (Young et al., 2003). Based on the model of early maladaptive schemas by Yang et al. (2003), maladaptive schemas are the inefficient cognitive schema to view self and others that have been mainly created in response to undesirable events in childhood and developed during the life. Yang (1996) hypothesized that early maladaptive schemas are the core of the personality disorders, temperament problems and many chronic disorders.

In the recent years, quality of life has been studied as one of the important outcomes of chronic diseases by the scholars and clinicians; so that evaluation of quality of Life is one of the essential aspects in clinical 
interventions and studies. Health therapists and researchers are especially interested in aspects of life that are more related to their health status, including mental and physical performance. The World Health Organization defines quality of life as people's perception of life situations and the relation to cultural context and life environmental value systems related to their objectives, criteria and interests (World Health Organization, 2008b). According to Van Maknsn (2007), quality of life is a broad term that covers a variety of concepts such as physical status, social cohesion, mental status, and health perception based on medical assessments, including health, performance of the patients, the effectiveness of treatment and, ultimately, the quality and efficiency of treatment and care.

Resilience is another variable in this study that is characterized by an individual's response to stressful life events or continuous exposure to stress (such as war and sexual exploitation) (Najmeh et al., 2012). Resilience enables people to use their adaptive skills and even changes the stressful situations into the learning or growth opportunities. It also helps people to control the stressful events by focusing the problem. It moderates the stress level in the stressful conditions. In fact, it is the positive dynamic process of adaptation and adjustment with the bitter experiences in life (Pourafzal et al., 2013). Resilience helps people to manage the stressful events and protect them from the mental disorders and life difficulties. Resilient people enjoy the high adaptation with the stressful events (Najmeh et al., 2012). Concerning the above-mentioned issues, it is clear that early maladaptive schemas, quality of life and resilience are affected a number of factors that should be investigated in order to improve the mental health of the people.

Ghazanfari (2006) conducted a study on the self-concept and mental health in eastern and western cultures. The results showed that the collective tendencies in Iranian girls play a key role in the formation of self-concept and mental health while individual self-esteem in Canadian girls plays an important role in mental health. Parto (2010) conducted a comparative study to investigate the mental health and life satisfaction and culture between two groups of young Iranians living in Canada and Iran. The results showed that all three variables of the mental health, life satisfaction, and culture of collectivism and individualism have the significant difference between immigrant and non-immigrant groups. Immigrants were significantly higher in terms of mental health, life satisfaction and culture of collectivism, but the differences between men and women migrants has not been confirmed.

Mohsen Aligh et al. (2009) in a study on the Taylor's pattern for psychosocial competence compared students in Iran and Lebanon with 172 Iranian and 146 Lebanese students. He concluded that in the Lebanese group, by increasing the external control and more positive feedback towards the world, proactive coping has been decreased. However, in Iranian group, high external control has been associated with decreasing the proactive coping. The relationship between the external locus of control and positive feedback towards the world in both groups was insignificant, but it was significantly higher in Iranian group compared to the Lebanese group. Danyr et al. (2000) reported that the correlation between self-esteem and well-being in developed countries is nearly 0.65 while in less developed countries is nearly 0.15 . This shows that when basic needs are satisfied, self-esteem is significant, but when basic needs are not satisfied, self-esteem is not significant and therefore not correlated with well-being.

Yancey et al. (2011) conducted a study on the role modeling, risk, and resilience in young people of California with 4010 of biracial 12-17 years old adolescents that were randomly selected using dialing. The results showed that ethnic balance among African Americans and whites of Latinos and Asians is higher. Ang et al. (2009) conducted a study to investigate cross-cultural invariance of the factor analysis of the stress list resulting from the academic expectations in two of the Malaysian and Canadian groups. They confirmed the cross-cultural validity of stress list resulting from the academic expectations and indicated that this kind of stress and the overall score of the stress in Malaysian group was significantly higher than the Canadian group. Meanwhile, Iran and Germany are two different countries in terms of social and cultural structures. Germany is a non-Islamic country, while Iran is an Islamic country and, naturally, this difference may affect the living conditions and lifestyle of the people and their families. As in previous studies the impact of culture on early maladaptive schemas, quality of life and resilience has less been studied, so this study aims to consider the important role of early maladaptive schemas variables, quality of life and resilience in a cross-cultural form. This study mainly seeks to answer this question: are the early maladaptive schemas, quality of life, and resilience in Iranian and German students different? 


\section{Method}

This was an applied study with descriptive causal-comparative data collection method, because the research variables were not manipulated and only the way demographic features of the statistical population were distributed and the better understanding of the prevalent conditions, were considered.

\subsection{Population, Sample, and Sampling Method}

The statistical population included all students at Karaj Azad University and all students at Saarland University in Saarbrucken, Germany. Multi-stage cluster sampling was used for the aim of this study so that four classes in technical-engineering faculties were selected from Saarland University and Karaj Azad University and questionnaires were distributed to the students attending the classes. Sample size included 200 students (100 in each university). Sample size included 100 students using the clausal-comparative method. For the experimental causal-comparative studies, the sample size consisted of 30 students in each group (Delavar, 2005).

\subsection{Measurement Instrument}

Questionnaires used in this study included:

1) Yang's Schema Questionnaire (short form with 75 items)

2) The World Health Organization's quality of Life questionnaire (short form)

3) Connor-Davidson's resilience scale

\subsubsection{Yang's Schema Questionnaire (Short Form with 75 Items) (YSQ-SF)}

This is a self-report instrument consists of a subset of 75 items that measures early maladaptive schemas (Young, 1998). In this questionnaire, the patients are asked to determine their degree of agreement on each item using a 6-point Likert scale. All five questions in the questionnaire measure a schema. If the average score of each item is higher than 3, the schema is inefficient (Nordahl et al., 2005).

The schemas that are measured in this questionnaire include:

- emotional deprivation (the belief that the one's emotional needs are not considered by others)

- abandonment/instability (the belief that others are an unreliable source of support and communication)

- mistrust/abuse (the belief the one can be hurt by others)

- social isolation/alienation (the belief that one is different from other people)

- defect/shame (the belief that the one is rejected by others because of the certain defects)

- failure (the belief that the one fails to communicate with others)

- dependence/incompetence (the belief that the one cannot do without necessary support of others)

- vulnerability to harm or illness (the belief that some crises are imminent)

- undeveloped self/enmeshment (the belief that the one needs high closeness in his or her relationships)

- obedience (the belief that one is forced to provide conditions for others in order to achieve what they need)

- sacrifice (the belief that their needs are satisfied without considering the needs of others)

- emotional inhibition (the belief that the emotional expression should be avoided)

- unrelenting criteria/extreme fault (faith in the strict criteria for individual performance)

- entitlement/nobility (the belief that the one is worthy of special privileges)

- self-control and self-discipline (the belief that one cannot control his impulses)

Lee et al. (1999) reported the favorable construct validity for the Australian version of the Young's Schema Questionnaire. The results of the factor analysis of the questionnaire supported thirteen Schema (Schmidt et al., 1995) and fifteen schemas (Lee et al., 1999) among the sixteen proposed schema. Fati et al. (2009) normalized the Persian version of this questionnaire on a sample of 513 Iranian students and reported the consistency within 18 subscales from 0.79 to 0.93 . Moreover, the re-test validity was obtained in the interval of 15 days for 18 schemas from 0.67 to 0.84 .

The results of the study on the psychometric properties of the short form of the Yang's questionnaire by Sadoughi et al. (2008) support the cross-cultural validity. 
In the present study, Cronbach's alpha coefficient for the initial schema was in the range of 0.89 to 0.74 ., indicating good internal consistency and reliability of short form of the early maladaptive Schema Questionnaire in this study.

\subsubsection{The World Health Organization's Quality of Life Questionnaire (Short Form)}

This questionnaire is a self-reported tool developed by the World Health Organization for evaluating the quality of life. The original test is a questionnaire containing 100 questions with 4 areas and 24 factors. Due to the length of the original test and the difficulty of completing it by some participants, the short version of this questionnaire called WHOQOL-BREF were provided by the WHO with 26 items. The first two questions consider a person's overall quality of life and health status and other 24 questions of the questionnaire examine four main dimensions, namely: physical health, mental health, social relationships, and environment health. Each question contains one of the 24 factors presented in the original questionnaire. Participants should respond the questions based on a 5-point Likert scale. This questionnaire is usually used for comparing the effectiveness and relative value of the different treatment, research, health policy, health service evaluation, treatment and improving the doctor-patient relationship.

Questions 1 and 2 in the questionnaire do not consider any of the four scales. Question 1 measures the person's overall life satisfaction and question 2 evaluates the overall health. Scores of these questions can be separately used. For obtaining the subscale score, the scores of all the expressions of the subscales are added and the resulting number is divided into number of the subscales. Thus, the average score for each subscale is obtained and questions 3, 4, 26 are reversely scored.

In order to evaluate the reliability, internal consistency based on the Cronbach's alpha coefficient was used. The Cronbach's alpha coefficient of the dimensions was reported as follows: physical health 0.87 , mental Health 0.74 , social relations 0.55 , and environmental health 0.74 . These values indicate good test reliability. This test has been normalized in Iran by Nejat et al. (2006) and Cronbach's alpha coefficient was reported as follows: healthy people 0.70 and patients 0.72 , mental health 0.73 and patients 0.70 , social relations 0.55 and patients 0.52 , environmental health 0.84 and patients 0.72 .

Differential credit: ability of the form short in distinguishing patients from normal individuals show the discriminant validity of the test. Furthermore, results of exploratory factor analysis indicate the favorable structure validity of this test. Persian validity: in order to ensure face validity, the questionnaire was twice translated into Persian and finally, a final version was prepared.

This version was twice translated separately into English. A copy of the English version was prepared and was compared with the original version. A significant difference between patients and healthy participants in four aspects indicated discriminant validity of the Persian version of the scale. In the present study, Cronbach's alpha coefficient for the questionnaire was 0.93 and for the dimensions was in the range of 0.75-0.91.

\subsubsection{Connor-Davidson's Resilience Scale}

This scale consisted of 25 items and was prepared by Connor and Davidson (2003) to measure the strength against pressure and threats. For each item, the five-point scale was considered from always true (1) o always false (0). Although this scale measures various aspects of resilience, gives an overall score.

Although, the results of internal consistency, test-retest reliability and convergent and divergent validity of the scale, have been sufficiently reported. Although the results of exploratory factor analysis have confirmed the five factors (competence/personal strength, self-confidence, personal instincts/tolerance of the negative emotions, positive emotions reception/safe relations, harness, spirituality) for resilience, they only reported the overall resilience score because the reliability and validity of the subscales have not been definitely confirmed (Connor \& Davidson, 2003).

Persian version of this scale has been prepared by Jokar (2007). In the study by Besharat et al. (2007), reliability and validity of the questionnaire were confirmed (quoted by Ranjbar et al., 2011). In the present study, the reliability of the questionnaire was tested using Cronbach's alpha coefficient as 0.87 . Therefore, this tool has a good reliability.

\subsection{Research Design}

Questionnaires were distributed as needed by referring to the selected classes and people who had the inclusion criteria for the study were selected. After providing explanations about the purpose of the study, questionnaires were individually administered with the presence of the observer on a sample group. In addition, subjects were informed that data collection is only for the aim of the study. Therefore, data will be confident in order to 
prevent the probable misuses. Some examples of German questionnaires were online available to the participants Then, the questionnaires were collected in due time, and preliminary analysis was provided. Inclusion criteria included being a student and exclusion criteria included age group over 40 and under 18 years old and not receiving psychiatric interventions over the one past year.

\section{Result}

Table 1. Distribution of the sample frequency by demographic variables

\begin{tabular}{cccc}
\hline Variable & Group & Frequency & Percentage \\
\hline Sex & Female & 136 & 68 \\
& Male & 64 & 32 \\
Country & Iran & 100 & 50 \\
& Germany & 100 & 50 \\
Age & $18-24$ & 131 & 65.5 \\
& $25-30$ & 53 & 26.5 \\
& $31-40$ & 15 & 7.5 \\
& Above 40 & 1 & 0.5 \\
\hline
\end{tabular}

According to Table 1, 32\% of the sample group was male and $68 \%$ were women. The highest frequency was observed for age group pf $18-24$ with $65.5 \%$ (131 people), and the lowest one for the age group over 40 with $0.5 \%$ (1 person).

Table 2. Descriptive findings of the variables in the two groups

\begin{tabular}{|c|c|c|c|c|}
\hline Group & Scale & Mean & $\begin{array}{c}\text { Standard Error of the } \\
\text { Mean }\end{array}$ & $\mathrm{SD}$ \\
\hline \multirow{3}{*}{ Iranian } & Early maladaptive schemas & 198.07 & 5.97 & 59.06 \\
\hline & Resilience & 61.27 & 1.34 & 13.69 \\
\hline & Quality of life & 81.65 & 1.44 & 14.21 \\
\hline \multirow{3}{*}{ German } & Early maladaptive schemas & 167.15 & 3.97 & 35.69 \\
\hline & Resilience & 63.34 & 1.25 & 10.76 \\
\hline & Quality of life & 84.36 & 1.30 & 10.87 \\
\hline
\end{tabular}

As shown in above table, in the early maladaptive schemas, Iranian students have the higher mean and in the quality of life and resilience, German students have a higher mean. In order to compare the early maladaptive schemas between two groups, multivariate analysis of variance (MANOVA) was used.

Table 3. Multivariate analysis of variance

\begin{tabular}{lccccccc}
\hline $\begin{array}{c}\text { Statistical indicator } \\
\text { of the trace }\end{array}$ & Test & Value & F & df Hypothesis & df Error & Sig & Trace Size \\
\hline \multirow{3}{*}{ Group differences } & Pillai's trace & 0.76 & 40.44 & 15 & 184 & 0.001 & 0.76 \\
& Wilks's lambda & 0.23 & 40.44 & 15 & 184 & 0.001 & 0.76 \\
& Hotelling's trace & 3.29 & 40.44 & 15 & 184 & 0.001 & 0.76 \\
& Roy's largest root & 3.29 & 40.44 & 15 & 184 & 0.001 & 0.76 \\
\hline
\end{tabular}


Table 3 shows the significant effect for the factor of "independent variable" using a multivariate variance analysis. This effect shows that there is a difference at least between one of the components of the early maladaptive schemas (Wilks Lambda $=0.23, \mathrm{p}<0.05$ ).

Table 4. One-way analysis of variance in the multivariate analysis of variance

\begin{tabular}{cccccccc}
\hline Statistical indicator of the variables & $\begin{array}{c}\text { Change } \\
\text { source }\end{array}$ & SS & df & F & Sig & $\begin{array}{c}\text { Trace } \\
\text { size }\end{array}$ & $\begin{array}{c}\text { Test } \\
\text { power }\end{array}$ \\
\hline emotional deprivation & group & 190.12 & 1 & 5.28 & 0.02 & 0.03 & 0.62 \\
abandonment & group & 1590.48 & 1 & 57.23 & 0.001 & 0.24 & 0.99 \\
mistrust & group & 15.68 & 1 & 0.44 & 0.51 & 0.01 & 0.10 \\
social isolation & group & 66.12 & 1 & 2.56 & 0.11 & 0.02 & 0.35 \\
Defect / shame & group & 0.08 & 1 & 0.01 & 0.98 & 0.01 & 0.05 \\
dependence & group & 1008.01 & 1 & 40.37 & 0.001 & 0.18 & 0.99 \\
vulnerability & group & 12.5 & 1 & 0.29 & 0.58 & 0.01 & 0.08 \\
enmeshment & group & 35.28 & 1 & 1.41 & 0.23 & 0.01 & 0.22 \\
Obedience & group & 411.84 & 1 & 16 & 0.001 & 0.08 & 0.97 \\
sacrifice & group & 18.61 & 1 & 0.25 & 0.61 & 0.01 & 0.08 \\
emotional inhibition & group & 4970.04 & 1 & 186.46 & 0.001 & 0.51 & 0.99 \\
unrelenting criteria & group & 212.18 & 1 & 9.44 & 0.002 & 0.05 & 0.86 \\
self-control & group & 3933.84 & 1 & 133.76 & 0.001 & 0.42 & 0.99 \\
entitlement & group & 3272.41 & 1 & 105.14 & 0.001 & 0.37 & 0.99 \\
failure & group & 524.88 & 1 & 19.96 & 0.001 & 0.10 & 0.99 \\
\hline
\end{tabular}

As shown in Table 4, the significance level obtained for most of the components of early maladaptive schemas is lower compared to the significance level of 0.004 obtained from the Bonferroni correction $(0.05$ significance level divided by 15 components of early maladaptive schemas). The effectiveness of the group differences or "practical significance" has been 0.74 for all components, namely, $74 \%$ of the total variance or individual differences in early maladaptive schemas has been related to the country or region. Moreover, the high statistical power of the statistical test in this study shows the proper rejection of the null hypothesis with the $99 \%$ probability.

In order to compare the resilience and quality of life in two groups, independent t-test was used.

Table 5. Comparison of means of the two groups in terms of resilience

\begin{tabular}{cccccccc}
\hline $\begin{array}{c}\text { Statistical } \\
\text { indicator of } \\
\text { groups }\end{array}$ & $\mathrm{N}$ & $\mathrm{M}$ & $\mathrm{SD}$ & $\begin{array}{c}\text { Mean } \\
\text { Differences }\end{array}$ & t Value & $\mathrm{df}$ & $\mathrm{Sig}$ \\
\hline Iranian & 100 & 61.27 & 13.69 & 2.07 & 1.18 & 198 & 0.23 \\
German & 100 & 63.34 & 10.76 & & & \\
\hline
\end{tabular}

As shown in Table 5, because t value (1.18) with the df of 198 is lower than the $t$ value in Table, and because the significance level of the test is 0.23 and higher than significance level of 0.05 , with the confidence level of $95 \%$, it can be said the difference between the scores of two groups is not significant. Therefore, the research $h$ hypothesis is rejected with the confidence level of $95 \%$ and the null hypothesis is confirmed. 
Table 6. Comparison of means of the two groups in terms of quality of life

\begin{tabular}{cccccccc}
\hline $\begin{array}{c}\text { Statistical } \\
\text { indicator of } \\
\text { groups }\end{array}$ & $\mathrm{N}$ & $\mathrm{M}$ & $\mathrm{SD}$ & $\begin{array}{c}\text { Mean } \\
\text { Differences }\end{array}$ & t Value & $\mathrm{df}$ & $\mathrm{Sig}$ \\
\hline Iranian & 100 & 81.65 & 14.21 & 2.71 & 1.51 & 198 & 0.13 \\
German & 100 & 84.36 & 10.87 & & & \\
\hline
\end{tabular}

As shown in Table 6, because $t$ value (1.51) with the df of 198 is lower than the $t$ value in Table, and because the significance level of the test is 0.13 and higher than significance level of 0.05 , with the confidence level of $95 \%$, it can be said the difference between the scores of two groups is not significant. Therefore, the research $h$ hypothesis is rejected with the confidence level of $95 \%$ and the null hypothesis is confirmed.

\section{Discussion and Conclusion}

Statistical analyses of the research hypothesis based on the significant difference showed the significant difference of the subscales of early maladaptive schemas of the Iranian and German students $(p<0.05)$. These findings are consistent with those of studies of Parto (2000), Mohsen Aligh (2009), Danyr et al. (2000), and Yancey et al. (2011). To explain these findings, the formation of early maladaptive schemas should be considered. Although a schema is not directly measured, but it can be measured through an analysis of personal life and observing the manner in which, people treat their talents and temperament.

This is observed when people present more details on their social behaviors in various social situations, life roles, and strategies.

Healthy schemas are formed when the baby's basic needs are met in a rich environment. This enables children to form a positive image about other people, themselves, and the world as a whole. Therefore, the formation of schemas is directly affected by the social and cultural conditions. Meanwhile, according to Yang, the people's thoughts or attitudes to life are different depending on the personality differences and educational pattern and are proved over time because of various personal experiences and consequently, the maladaptive schemas are formed.

This conceptual framework consists of the beliefs that result in different attitudes to the others, life, educational pattern, job and so on. This difference was also observed between two Iranian and German culture. There was no significant difference between Iranian and German students in term of resiliency $(p>0.05)$. These findings are not consistent with those of Yancey et al. (2011). Yancey et al. showed that resilience among African Americans and whites of Latinos and Asians is higher. For explaining these findings, new findings within the scope of resiliency should be considered. The new findings on resilience are regarded as a multidimensional construct of temperament variables, such as temperament and personality with special skills like problem-solving skills. Therefore, resilience is not a mere genetic phenomenon of the certain people.

In fact, the resilience is the inherent capacity of the people that can lead to self- reform and change. Resilience that is also called resistance to the stress or post-traumatic growth is considered along a continuum with varying degrees of resistance to the psychological injuries. Therefore, it is beyond escaping the stresses and life difficulties and is the growth, adaptation or achieving the balance after imbalance in the previous balanced situation. So, however, there is a little difference between the Iranian and German students in terms of resiliency, this construct is influenced by specific individual experiences rather than cultural situation.

There was not also a significant difference in terms of the quality of life of Iranian and German students $(\mathrm{p}>0.05)$. This is not consistent with that of Parto (2000). Parto (2000) conducted a study to compare the mental health and life satisfaction and culture between two groups of young Iranians living in Canada and Iran. The results showed that all three variables of mental health, life satisfaction, and culture of collectivism and individualism show the significant difference in two groups of immigrant and non-immigrant.

To confirm this it should be said that quality of life in this study has not been shown and it has been measured based on the self-report of the people. Therefore, quality of life in this way is an overall evaluation of the living conditions of the people. Quality of life is a subjective concept and personal perceptions about quality of life of are the most practical judgements. Bond (1997) states that the quality of life of each person is what he or she explains. The one's identification and assessment of life and satisfaction with social, physical, mental, and emotional health status is not based on his or her physical limitations. Kohli et al. (2005) deal with quality of life such as a general feeling of improvement including happiness and life satisfaction. 
On the other hand, since the research sample in Iranian group was selected from among the Azad University and these students are of higher welfare compared to other Iranian students, there was no significant difference between the quality of life of Iranian and German students. Due to the negative effects of the social and cultural issues in Iran on formation of the early maladaptive schemas and the fact that schemas usually lead to biases in the interpretations of the realities that are manifested in the form of misunderstandings, closed attitudes, false logic and unrealistic goals and expectations, the Iranian society requires information on the nature and function of the schemata improve the public health.

\section{References}

Ahi, G. (2005). Normalization of the Young's Schema Questionnaire short form (SQ-SF) among students of Tehran University (Master's thesis). Faculty of Psychology and Educational Sciences, Allameh Tabatabaei University.

American Pychiatyic Associati (APA). (2000). Diagnosis and Statistical Manualof Mental Disorders Text Revision (DSM-IV-TR) (4th ed.). Washington, DC., American Psychiatric Association, Publishing.

Anderson, K. et al. (2006). A comparison of maladaptive schema in treatment-seeking obese adults and normal-weight control subject. Journal of psycjosomatic research, 60, $245-252$. http://dx.doi.org/10.1016/j.jpsychores.2005.08.002

Anmuth, L. M. (2011). Early Maladaptive Schemas and Negative Life Events in the Prediction of Depression and Anxiety. Master of Arts thesis in Clinical Mental Health Counseling, Rowan University.

Arntz, A., Klokman, J., \& Sieswerda, S. (2005). An experimental test of the schema mode model of borderline personality disorder. $J$ Behav Ther Exp Psychiatry, 36(3), 226-239. http://dx.doi.org/10.1016/j.jbtep.2005.05.005

Asgari, H., \& Roshani, H. (2007). Emotion recognition, clinical and social aspects. Tehran: Fahangan.

Calvete, E., Estévez, A., López de Arroyabe, E., \& Ruiz, P. (2005). The Schema Questionnaire-Short Form. Structure and relationship with automatic thoughts and symptoms of affective disorders. European Journal of Psychological Assessment, 21, 90-99. http://dx.doi.org/10.1027/1015-5759.21.2.90

Dadsetan, P. (2011). Developmental psychopathology from childhood to adulthood (Vol. I). Tehran: Samt.

Dozois, D. J. A., Martin, R. A., \& Bieling, P. J. (2009). Early malaeaptive schemas Factor Analysis of the Young Schema Questionnaire-Short Form in a Nonclinical Iranian Sample. IJPCP, 14(2), 214-219.

Hoksema, S. H. (2008). Abnormal psychology: Media and reaserch update. Megrawhill pub, 192-230.

Kameli, Z. (2010). The effectiveness of cognitive group therapy based on schema on modification early maladaptive schemas among orphan and dysfunctional parenting adolescents girls.

Kaplan, H. I. et al. (1994). Summary of behavioral sciences psychiatry-clinical sciences psychiatry (N. Pourafkari, Trans., Vol. II). Tehran: Shahrab.

Kaplan, H. I. et al. (2007). Summary of psychiatry (H. Rafiei, \& F. Rezaei, Trans.). Tehran: Arjomand.

Rayner, J. S. (2008). The relationship between patterns of relating and early maladaptive schemas. Presented for the doctor of philosophy degree the university of Mississippi.

Schmidt, N. B., Joiner, T. E., Young, J. E., \& Telch, M. J. (1995). The Schema Questionnair: Investigation of psychometric properties andthe hierarchical structure of a measure of maladaptive schemata. Cognitive Therapy and Research, 19(3), 295-321. http://dx.doi.org/10.1007/BF02230402

Schmidt, N. B., \& Joiner, T. E. (2004). Global maladaptive schema: Negative life events and psychological distress. Journal of psychopathology and behavioral assessment, $26,65-71$. http://dx.doi.org/10.1023/B:JOBA.0000007457.95008.d2

Sharf, R. (2011). Theories of psychotherapy and counseling. Tehran: Ravan.

Sharifi, A. (2010). The relationship between personality types and early maladaptive schemas. Master's thesis on career counseling, Faculty of Psychology and Educational Sciences, Allameh Tabatabaei University.

Welburn, K., Coristine, M., Dagg, P., Pontefraet, A., \& Jordan, S. (2002). The schema question-short form: Factor analysis and relationship between schemas and symptoms. Cognitive therapy and research, 26, 519-530. http://dx.doi.org/10.1023/A:1016231902020 
Young, J. (1950). Cognitive therapy of personality disorders: Schemas-centered approach (A. Sahebi, \& H. Hamidpour, Trans.). Tehran: Arjomand.

Young, J. E. (1995). Cognitive therapy for personality disorders: A schemafocused approach. Sarasota FL: Professional Resource Exchange.

Young, J. E. (1999). Cognitive therapy for personality disorders: A schemafocused approach (rev. ed.). Sarasota, FL: Professional Resources Press.

Young, J. E., \& Behary, W. T. (1998). Schema-focused therapy for personality disorders. In W. A. Tarnier, \& G. Haddock (Eds.), Treating complex cases: The cognitive-behavioral therapy approach (pp. 340-376). Chichester: Wiley.

Young, J. E., \& Brown, G. (1990). Young Schema Questionnarie (2nd ed.). New York: cognitive therapy center of New York.

Young, J. E., \& Kolosko, J. S. (1994). Reinventing your life. New York: Plume Books.

Young, J. E., Kolosko, J. S., \& Weishar, M. E. (2003). Schema therapy: A Partitioers guide. New York: Guilford press.

Young, J. et al. (1950). Therapy Schema (guidance for clinicians) (H. Hamidpour, \& Z. Andouz, Trans.). Tehran: Arjomand.

Young, J., \& Martin, R. (2010). Schema therapy. In K. S. Dobson (Ed.), Handbook of cognitive-behavioral therapies (pp. 317-346). New York: The Guilford press.

\section{Copyrights}

Copyright for this article is retained by the author(s), with first publication rights granted to the journal.

This is an open-access article distributed under the terms and conditions of the Creative Commons Attribution license (http://creativecommons.org/licenses/by/3.0/). 